\title{
Breaking BIM: A Transformative Design Methodology for Building Information Modeling
}

SETH MCDOWELL

University of Virginia
Building Information Modeling (BIM) is no longer just a tool for project delivery and production. It is now a critical, precise tool for design and analysis. This research on "Breaking BIM" seeks to identify new relationships between design processes and BIM that leverage the computational resources for design objectives. The paper outlines three approaches to breaking the constraints of BIM with more intuitive workflows for design. These include associative modules, conceptual massing, and component hacking. The work here is a summary of four years of experiments in dissecting Autodesk's Revit. The explorations occurred as teaching activities and within my own design practice.

A methodology will be described for designing through relational objects that identifies BIM as a process of transformative design. With a transformative design methodology, the designer is given a default typology, and must deconstruct or reconfigure the typology to establish a new type. This process of object transformation characterizes design customization strategies in common BIM platforms like Revit.

\section{INTRODUCTION}

It is undeniable that Building Information Modeling (BIM) has aided architects with production operations, yet BIM platforms still struggle with the intuitive, messy design process that characterizes the work of creative, progressive architects. It is a lot easier to conceptualize and study design within the loose, mostly uncoordinated framework of surface modeling programs like Rhino or SketchUp or for nostalgic designers with physical models and sketches. What I would like to emphasize and exhibit in this paper is that BIM is not just a production or project delivery tool. Nor is BIM purely limited to the pragmatic buzz words that usually accompany the acronym, such as "integration," "collaboration," "optimization," or "simulation." My objective in "Breaking BIM" is to identify new relationships between design processes and Building Information Modeling that leverage the computational resources for design objectives.

Examining BIM as a design platform is a challenge because as the built-in assumptions and efficiencies increase in the tool, the ability to experiment and discover new possibilities decrease. The Bauhaus educator Josef Albers highlighted in his teaching that "assumptions can blind us to the real possibilities." ${ }^{1}$ Thus, it is paramount to link BIM with concep- tual, formal exercises that encourage architects to critically evaluate, test, and disrupt these software assumptions with design objectives. If this does not happen-if we cannot find a way to establish formal, spatial, and conceptual exploration within such a robust, yet structured digital platform - then we are not operating as designers within the twenty-first century. A 2014 survey by the software training website Black Spectacles identified that over $70 \%$ of job postings for architects required proficiency in Revit. ${ }^{2}$ It is now inevitable that designers wi II encounter BIM in future practice, and if they do not have the patience to hack and customize the default assumptions to their own agenda, then we will be heading toward a highly depressing architectural future.

Overcoming the challenges and assumptions surrounding BIM as a design tool are inhibited by a paranoia about control. Most of us working in architecture today have emerged from an age when you were taught from the very first foundation studio or drawing course to construct space, material, and form with the elements of points, lines, and planes. Beginning with these elemental ingredients, the designer has control of the essential DNA of an object. It is easy to control and manipulate points, lines, and planes. When working in a disassociated picture plane, you can work extremely intuitively, and the only constraints are what your mind or eye impose upon the blank sheet. There is freedom to sketch, draft, or model whatever shape you please.

Perhaps the ease and freedom we have with the unconstrained pencil has formulated our direct association between design and customization. We often call something "designed" only when it has been rendered in a way that is different from the conventional typology. Straying from traditional typologies is easy when it is a matter of moving a point or line. It is easy to customize when you are working within an untethered environment and you can intuitively compose without consequences. What frightens most trained under these precepts is that with BIM the elemental construction device is no longer the point, line, or plane. In BIM, the element is the wall, the floor, the door, the window, or some other object. In BIM, design occurs with parametric objects, not abstract points and lines, and that is terrifying to those who are comfortable with constructing from abstraction.

This methodology of designing through relational objects does not mean control is surrendered. Rather, design control 


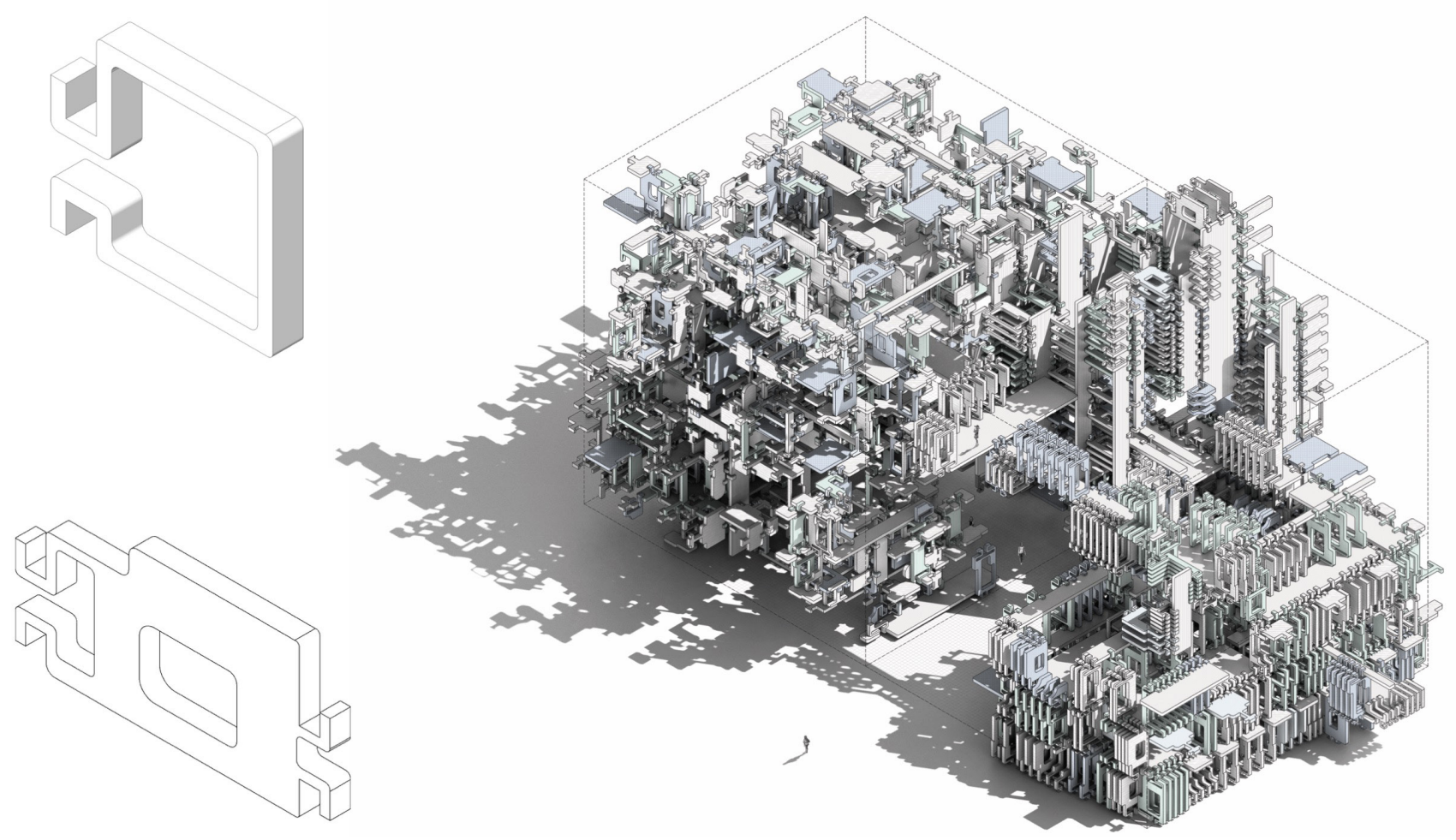

Figure 1: Conceptual Revit studies for 2015 Architecture League Prize Exhibition.

simply changes from creational control to transformative control. With creational control, the designer constructs the product from scratch using the DNA of points, lines, and planes. With transformative control, the designer is given a default typology, and must deconstruct or reconfigure the typology to establish a new type. Working with BIM is largely a transformative design methodology in which you are given the default object embedded with a set of default parameters, and then you must edit and transform these default assumptions to achieve customization.

\section{METHODS AND OBJECTIVES: A TRANSFORMATIVE DESIGN METHODOLOGY FOR BIM}

To Break BIM and explore it for design objectives rather than purely for production opportunities, it is best to have the mindset of a tinkerer. In BIM you inherit a collection of default components or objects, and to achieve specific design objectives you must hack these objects. This establishes the first theoretical objective for Breaking BIM: to approach design as a transformative act which transforms the "default," "basic," or "generic" archetype into an exception.

The act of object transformation is nothing new to architecture and owes homage to Marcel Duchamp's experiments with the "ready-made." The tinkering operations Duchamp and his cohorts in Dadaism performed on ubiquitous, visually mute objects of industrialization echo the default-component hacking approach to BIM. Duchamp's 1917 Fountain, a urinal that is now the most referenced art piece from the twentieth century, positioned the everyday object as a raw material in a new environment. Fountain extracted the mundane, utilitarian urinal from the bounds of a prescribed function and placed the object within a foreign context: the gallery. It was an act of transformational creation.

What we learn from Duchamp is that the mundane object can be elevated through a few simple operations. Relocating the object to a new context disassociates the object from its initial utility and parameters. Reorientation also works to dismantle the default assumptions for the object, as Duchamp's initial Fountain was displayed on a pedestal, not a wall, which required it to sit horizontally. Finally, Duchamp signed the object "R. Mutt," establishing its new typology as an authored work of art. This whole act of object transformation is paralleled in a BIM program like Revit when one takes a default object, like a door, opens it in the family editing environment (a new isolated context), and begins to redefine the hosting element from wall to floor. After these virtual object alterations, label the door with a name, like "R. Mutt" and a new BIM family is created.

BIM produces a virtual building. Fundamentally, it repositions architectural design from an exercise in representation to an exercise in simulation. The architect now constructs a model rather than producing illustrations. This situation prompts the second theoretical objective for "Breaking BIM": to employ the logics of construction as a design operative. In other words, BIM allows the designer to think like a builder and to immediately learn and react to the spatial consequences of 


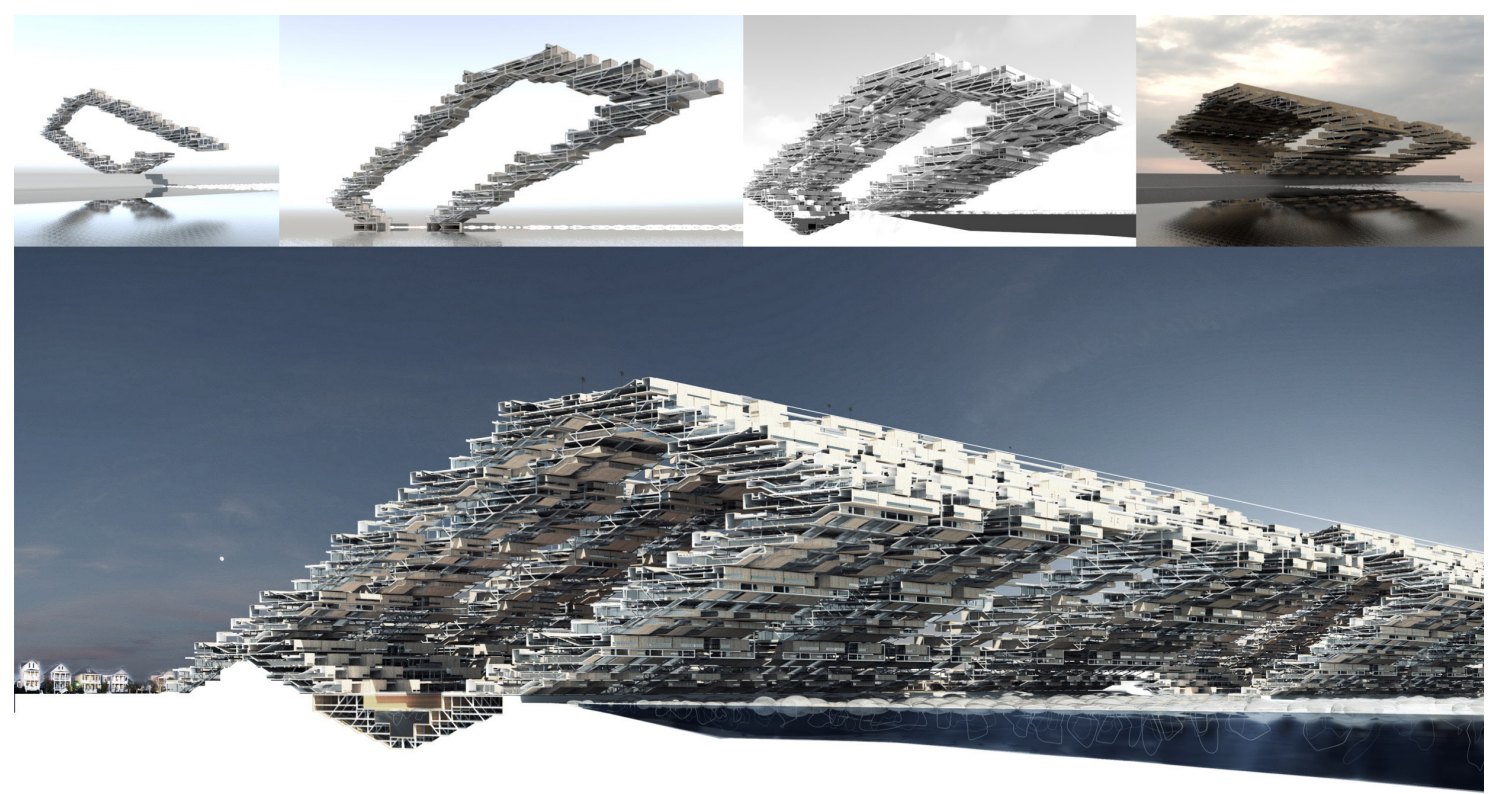

constructing. In traditional drafting programs or 3D modeling programs, there is segregation between the detailing and the spatial consequences. With BIM, however, the designer can immediately simulate the spatial consequences of construction and material decisions.

What you quickly discover when working within a building model is that much of your time is spent modeling virtual connections, customizing material parameters, and positioning building elements. In this way, the architect works very much like a builder-creating scaffolding, jigs, and parameters that allow for this positioning, connectivity, and finishing of building elements. The virtual building model creates an environment that makes it impossible to fake it-a resolution many lazy designers resort to when drawing or modeling in an untethered, uncoordinated environment.

This objective to employ the logics of construction as a design operation extends beyond the fact that architects are now working within a virtual model rather than two-dimensional picture planes. I am interested in using acts of construction as generative operations. Instead of beginning design with a set of compositional ideas that later get rationalized for construction purposes, construction logics can allow a design strategy to emerge in an indeterminate manner from methods of assembly. This construction methodology will be more apparent in the approaches that follow.

\section{RESULTS: BREAKING BIM}

The following approaches to break the constraints of BIM are a summary of four years of experiments in dissecting Autodesk's Revit. The explorations occurred as teaching activities and within my own design practice. The teaching explorations were executed in a third-year undergraduate foundation studio. The curricular objective for this studio is to introduce students to their first substantial building design project that contains sectional and programmatic complexity. It is a daunting task, as students are encroaching on new territory both with BIM and with the design project.

\section{APPROACH 1-ASSOCIATIVE MODULES}

The associative module approach to disrupt BIM begins as an analog, physical modeling exercise. The process is based on Revit's generic component family, which works well with a modular approach to design. The conceptual exercise is to construct a puzzle without a picture-meaning, without any predetermined form or shape, encode a collection of parts or modules with a relational method of assembly. This could entail shaping the module for nesting configurations or a void operation that enables intersection. This encoded interface informs, if not dictates, a method of assembly. It is an associative joint that enables relationships to be established with the other modules. A three-dimensional puzzle is generated as the construction logic plays out. Iterations can be quickly generated by taking the three-dimensional puzzle apart and reassembling it in a different way. There is a constant feedback loop that encourages the module to be altered or refined to allow for improved outcomes. The modules can be identical or completely varied, depending on design objectives.

While the exercise may begin as a physical modeling exercise, it should quickly be translated to Revit using generic components-a Revit family that has no specific identity or default parameters. The generic component family is stripped of assumptions and encourages maximum freedom and customization. Within the family interface, parameters can be built into the module so that it can expand, shrink, 


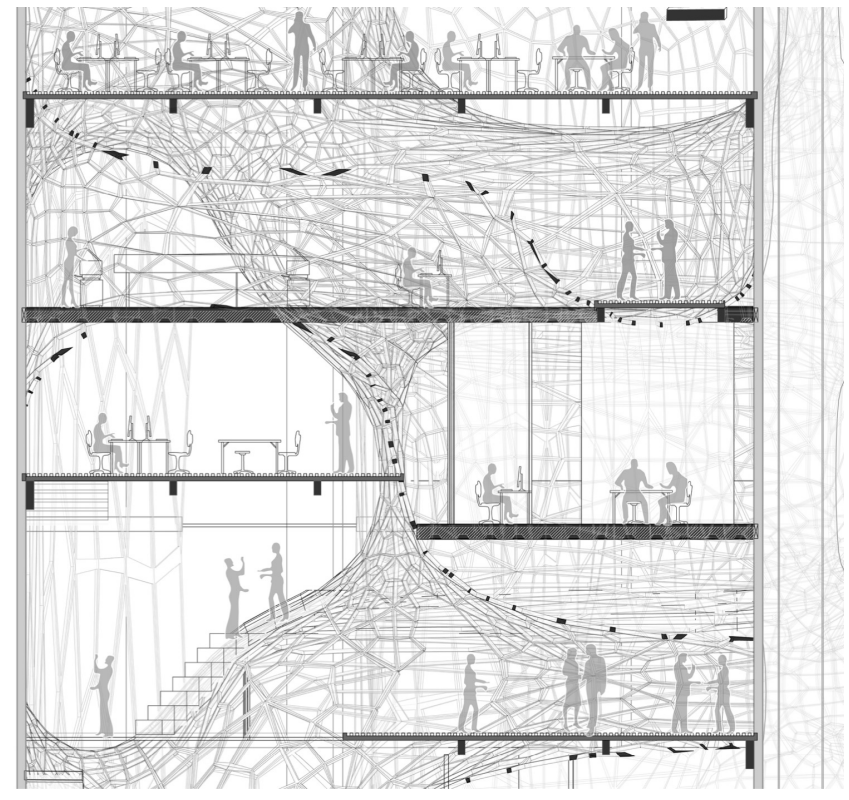

Figure 3: Dixi Wu's undulating "monster" created by a workflow between Grasshopper and Revit's conceptual massing.

and transform based on variables. This workflow contributes to an extremely intuitive, flexible working process that can verge on a game of BIM Tetris or Jenga. It is a playful way to explore and sketch in Revit, which is the goal. (Figure 1)

This associative module approach can extend beyond the scale of components and be interpreted into urban organization strategies or building modules. In a project called Drift City the module was translated to the scale of a city sector in a speculative city (Figure 2). Here, modules are nested inside of modules and parameters are constructed to allow for variations in density and program. Regardless of scale or function, beginning with a generic component family allows the designer to sketch with a simplified set of modules that can increase in complexity as the project develops. Generally, these modules start as abstract solids that are later translated into building components like walls, floors, roofs, or structure.

In evaluating this process against my two objectives for breaking BIM, both goals are accomplished. First, by beginning design with a generic component, the immediate protocol is to transform the default generic object into a custom module. Generic Components are one of the easiest families to work with in Revit due to their lack of definition. Therefore, they lend themselves to exploratory exercises. Second, the whole exercise is motivated by construction or assemblage. It is an additive process where form must be constructed rather than sculpted.

\section{APPROACH 2-CONCEPTUAL MASSING}

If the first approach to break BIM is a bottom-up approach that follows an additive process, the second approach reverses this and is situated as a top-down massing strategy. This process is aided by Revit's conceptual massing family which, like generic components, creates a stripped-down environment with very few default assumptions; it is simply a Cartesian space of $X, Y, Z$ construction planes, much like what we find in Rhino. The modeling operations are equally primitive and create form by means of extrusion, lofting, sweeping, and blending. This uninhibited interface encourages play similarly to more intuitive programs like Rhino, SketchUp, or Formlt. The advantage of three-dimensional sketching within Revit's conceptual massing family as opposed to these external programs is that geometry inherent to Revit always tends to be more flexible to work with throughout the building development process. Massing inherent to Revit also simplifies the workflow and allows for extremely fast transitions between concept sketching and building development.

Conceptual massing disproves the criticism claiming that complex, curvilinear form is impossible in Revit. Dixi Wu's project for the NYC Urban Design Institute reveals that conceptual massing can generate extremely seductive form compatible with Revit's building components (Figure 3). Wu developed an undulating "monster" as a conceptual massing family, aided by some mesh relaxation definitions in Grasshopper, and then translated the surface's U/V grid into a structural grid using a Revit curtain system.

This approach to sculpting form is a top-down approach because it is generally focused on building envelope. Conceptual massing essentially constructs a skeleton or ghost that will host the building elements. It allows for quick manipulations in form, without having to adjust every little part of the building. Efficiency is created from the associative relationship between the building elements (walls, floors, and roofs) and the conceptual mass. At any stage of the project's development you can return to the mass and make global, massing alterations - then reattach the building elements to the host to enable local updates.

The conceptual massing approach can also allow for the inclusion of more advanced tools like adaptive components, where complex surfaces of the mass can be panelized with custom, adaptive panels that respond to grid configurations. This was explored in an experiment in which a curvilinear surface was panelized with a three-dimensional adaptive component that conformed to the grid sizing (Figure 4). This process allows the designer to link global customization of form with local customization of parts.

In evaluating this approach through the viewpoint of my two theoretical objectives, I find it less successful than the associative modules route largely because of the fixation on form making. While extreme customization is achieved, it is generally accomplished through creational control rather than a transformational logic. Conceptual massing allows the 


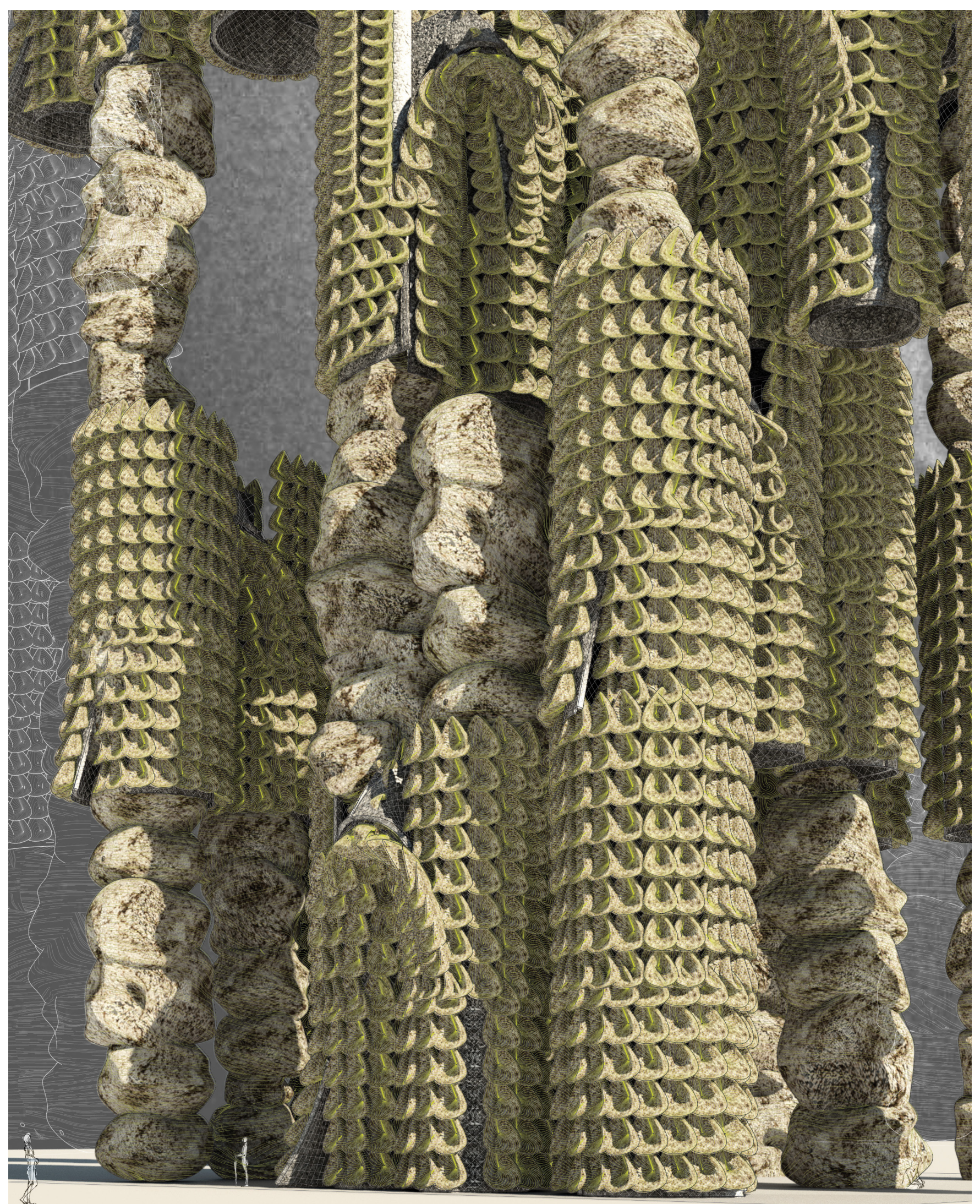

Figure 4: The combination of conceptual massing and adaptive components for a sculptural study. 


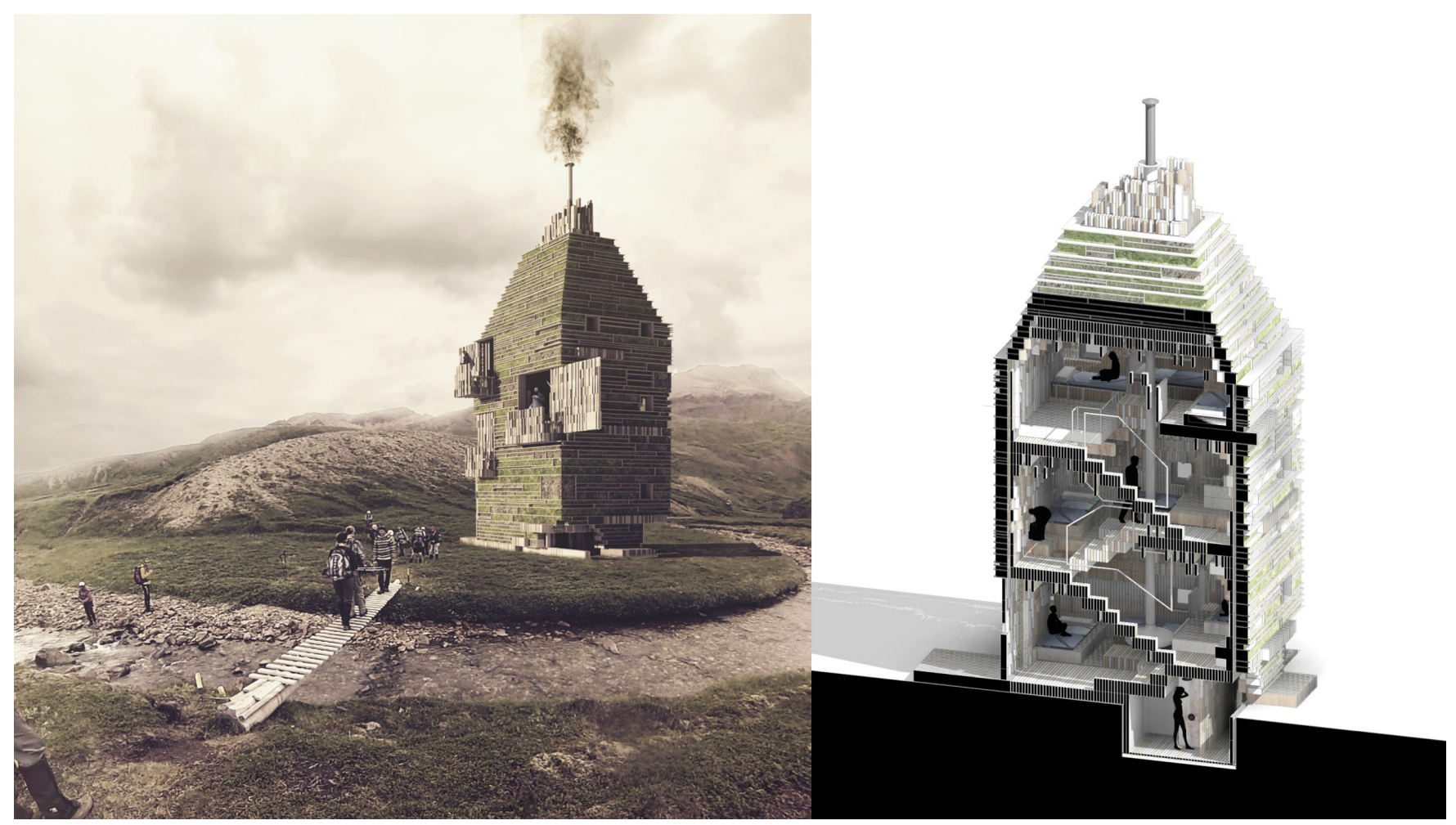

Figure 5: Component hacking approach, every building element is constructed from a curtain wall tool.

designer to go back to the basic elements of point, line, and plane, which are great for enabling creative freedom, but this diverges from my objective to explore BIM as a transformative act. The workflow also fails to support my objective to deploy construction operations as a design process. In conceptual massing, form is created and then rationalized, which contradicts my desire to use assembly systems to generate form. While this approach to break the constraints of BIM is successful in the sense that it provides an uninhibited method for working intuitively within the Revit platform, it is unsuccessful in accomplishing my own theoretical objectives.

\section{APPROACH 3-COMPONENT HACKING}

Component hacking is the approach for breaking BIM that most directly relates to object transformation. While the previous two approaches build custom families from scratch, component hacking takes a default component, such as a curtain wall, and manipulates it. This is the Duchamp method for breaking BIM, where an object's use, orientation, and context are completely redefined. This requires a more systematic way of designing that is less like blissful sketching, and more surgical. It requires digging into the DNA of these default BIM components and reorganizing how they work.

The curtain wall is the most useful component to examine through hacking. Essentially, a curtain wall in Revit is a vertical plane that can be divided into panels. The grid that divides the plane can also host mullions, creating a panel and frame system. At first, because of all the elements nested into the curtain wall, it seems like a cumbersome component to work with. However, when you start to break it down, it is an extremely robust and versatile element that can be reconfigured in many ways. In a project called Sod-Lam, I constrained myself to build everything in the model with curtain wall components. Stairs, windows, doors, even beds, became part of the curtain wall system (Figure 5). The result was two layers of laminated walls-one creating an internal shell of wood members, and one creating an external shell of sod panels. Using the curtain wall component in this way allowed for systematic design studies.

Creating this project solely based on a redefined Revit component was an interesting intellectual exercise, but admittedly it creates some management issues. The lesson I have learned with Revit is that customization tends to create a ripple effect of new issues to coordinate. For instance, when using a curtain wall for a stair, you do not have the benefits of calculation tools to ensure rise and run are within acceptable ranges. You also lose simple annotation tags that are inherent to stair families. Thus, many of BIM's efficiencies may get dismantled in this process of object transformation-a risk that must be evaluated.

In assessing this approach for breaking BIM relative to my theoretical objectives, I find it to be extremely successful. More than the other two methods, component hacking is deeply rooted in object transformation. The act of transformation forces the designer to examine the assumptions of these objects. When disassembling components like a door or 
curtain wall, you learn a lot about the gadgetry that enables it to work. Again, this is like tinkering. If you take apart your car engine, you learn a lot about mechanics. Then, when you put it back together, you might accidentally create a new, improved machine-or at least a Frankenstein.

\section{COLLAPSING THE HIERARCHIES OF MAKING}

Before the cyanotype, John Herschel's 1842 technological milestone, an incredible amount of labor in architectural design was expended on copying drawings. The cyanotype, or blueprint, created a photographic process that enabled the inexpensive duplication of images. This apparatus had a dramatic impact on architecture practice, reducing staffs by $60 \%$ - a shocking statistic that highlights the amount of routine labor embedded in the process of architectural design. The desire to eliminate tedious, repetitive tasks has since been a primary motivation for the evolution of architectural tools for visualizing buildings-progressing from T-Square to Pin-bar drafting to Computer Aided Drafting, and more recently to Building Product Models, Building Information Models, and parametric modeling. In theory, the reduction of tedious representation tasks reduces errors and miscommunications in the architect's work.

During this evolution, the strategy shifted from physical duplication techniques to information management. Pin-bar drafting, a short-lived manual drafting method in the 1980s, involved layering sheets of information to improve efficiencies. Similarly, early CAD programs layered data groups in a digital environment-isolating shared, global data from localized data specific to one drawing. These rudimentary information management systems were structured around external references that could be shared across multiple drawings, while more view-specific information, like annotation or furnishings, could be layered upon the global reference. If a dimensional change occurred, it could be made on the base layer and immediately be reflected in all drawings referencing the base layer. With these fundamental strategies for information management, the goal became clear: because architectural design is a very unstable process, the tools for visualizing and describing design intentions need to be intelligent and coordinated to accommodate inevitable and constant modification.

Then enters Building Information Modeling-a seemingly intelligent process for constructing virtual models that are both coordinated and computable. BIM integrates 3D visualizations and 2D representations. Because BIM is a live environment, a change in plan is immediately reflected in elevation. BIM is injected with information tags that enable quick itemization of every element in the building. Even 4D data like time, cost, and energy can be simulated within this information modeling system for architecture.

$\mathrm{BIM}$ is a beast that simultaneously requires higher-level knowledge and routine labor. With BIM we have to shed the typical hierarchies of creative production. The visionary and the draftsperson must work as one entity because design occurs through virtual construction. It is for this reason that it is critical to take BIM seriously in academia-because the designers of the future are not going to be sitting in their corner offices, painting with watercolors or sketching with charcoal or gluing chipboard together, only to hand it off to an apprentice to execute. If that continues to be the format for design, we have missed our opportunity to reap the benefits of Building Information Modeling.

\section{ENDNOTES}

1 Albers, Josef. "Search Versus Re-Search: Three Lectures." Lecture, Trinity College, Hartford, CT, April 1965.

2 Quirk, Vanessa. "Want to Land a Job at One of the Top 50 Architecture Firms? Here Are the Skills You Need to Have..." ArchDaily. 15 July 2014. 